\title{
Can bedside patient-reported numbness predict postoperative ambulation ability for total knee arthroplasty patients with nerve block catheters?
}

\author{
Seshadri C. Mudumbai ${ }^{1,2}$, Toni Ganaway ${ }^{1,2}$, T. Edward Kim ${ }^{1,2}$, \\ Steven K. Howard ${ }^{1,2}$, Nicholas J. Giori ${ }^{3,4}$, Cynthia Shum ${ }^{2}$, and \\ Edward R. Mariano ${ }^{1,2}$ \\ ${ }^{1}$ Department of Anesthesiology, Perioperative and Pain Medicine, Stanford University School of Medicine, \\ ${ }^{2}$ Anesthesiology and Perioperative Care Service, Veterans Affairs Palo Alto Health Care System, Department of \\ Orthopaedic Surgery, Stanford University School of Medicine, ${ }^{4}$ Surgical Service, Orthopaedic Surgery Section, \\ Veterans Affairs Palo Alto Health Care System, CA, USA
}

Background: Adductor canal catheters offer advantages over femoral nerve catheters for knee replacement patients because they produce less quadriceps muscle weakness; however, applying adductor canal catheters in bedside clinical practice remains challenging. There is currently no patient-reported outcome that accurately predicts patients' physical function after knee replacement. The present study evaluates the validity of a relatively new patient-reported outcome, i.e., a numbness score obtained using a numeric rating scale, and assesses its predictive value on postoperative ambulation.

Methods: We conducted a retrospective cohort study pooling data from two previously-published clinical trials using identical research methodologies. Both studies recruited patients undergoing knee replacement; one studied adductor canal catheters while the other studied femoral nerve catheters. Our primary outcome was patient-reported numbness scores on postoperative day 1 . We also examined postoperative day 1 ambulation distance and its association with postoperative numbness using linear regression, adjusting for age, body mass index, and physical status.

Results: Data from 94 subjects were included (femoral subjects, $\mathrm{n}=46$; adductor canal subjects, $\mathrm{n}=48$ ). Adductor canal patients reported decreased numbness (median $\left[10^{\text {th }}-90^{\text {th }}\right.$ percentiles]) compared to femoral patients $(0[0-5]$ vs. 4 [0-10], $\mathrm{P}=0.001)$. Adductor canal patients also ambulated seven times further on postoperative day 1 relative to femoral patients. There was a significant association between postoperative day 1 total ambulation distance and numbness (Beta $=-2.6 ; 95 \%$ CI: $-4.5,-0.8, \mathrm{P}=0.01)$ with $\mathrm{R}^{2}=0.1$.

Conclusions: Adductor canal catheters facilitate improved early ambulation and produce less patient-reported numbness after knee replacement, but the correlation between these two variables is weak.

Key Words: Adductor canal block, Ambulation, Femoral nerve block, NumbnessPerineural catheter, Total knee arthroplasty.

Corresponding author: Edward R. Mariano, M.D., M.A.S.

Anesthesiology and Perioperative Care Service, VA Palo Alto Health Care System, 3801 Miranda Avenue (112A), Palo Alto, CA 94304, USA

Tel: 1-650-849-0254, Fax: 1-650-852-3423

E-mail: emariano@stanford.edu

ORCID: http://orcid.org/0000-0003-2735-248X

Received: June 3, 2015. Revised: July 8, 2015. Accepted: July 13, 2015.

Korean J Anesthesiol 2016 February 69(1): 32-36

http://dx.doi.org/10.4097/kjae.2016.69.1.32

(c) This is an open-access article distributed under the terms of the Creative Commons Attribution Non-Commercial License (http://creativecommons.org/ licenses/by-nc/4.0/), which permits unrestricted non-commercial use, distribution, and reproduction in any medium, provided the original work is properly cited. 


\section{Introduction}

For patients undergoing total knee arthroplasty (TKA), evidence supports the use of continuous peripheral nerve block (CPNB) techniques with femoral or saphenous perineural local anesthetic infusions as part of a perioperative multimodal analgesic regimen [1-3]. Although CPNB offers clear analgesic advantages with selectivity for the operative extremity by targeting specific nerve distributions, there is rising concern over the quadriceps weakness produced by femoral nerve blocks and the potential risk of falls [4,5]. More recently, the adductor canal catheter (ACC) technique [6] has emerged as an alternative to the femoral nerve catheter (FNC) for TKA patients, because it better preserves quadriceps motor function while still providing effective pain relief $[7,8]$, and may facilitate improved early ambulation after surgery [9].

While previous studies have assessed quadriceps strength in terms of maximum voluntary isometric contraction [10-12], this method requires the use of a dynamometer and is not routinely part of clinical bedside assessment. Clinical assessments commonly rely on physical examination and subjective patientreported outcomes (e.g., numeric ratings of pain). Although the use of numeric rating scales (NRS) of patient-reported numbness has been reported previously $[13,14]$, this measure has not yet been correlated with physical function and also remains to be evaluated as a predictor of postoperative ambulation. A patient who self-reports numbness of the quadriceps muscle on the operative extremity after TKA may be more reluctant to participate in physical therapy due to fear of falling. Given the expected increase in TKA prevalence over the next 10 to 15 years, due to an aging population and rising prevalence of osteoarthritis [15], there is a need to critically evaluate assessment techniques and incorporate patient-reported outcomes as appropriate.

We designed this study to test the hypothesis that TKA patients with an ACC will exhibit lower self-report numbness scores on an NRS compared to patients with an FNC. We also explored the potential correlation between patient-reported numbness and ambulation distance on postoperative day (POD) 1.

\section{Materials and Methods}

After receiving Institutional Review Board approval with waiver of informed consent (Stanford University School of Medicine, Stanford, CA, USA), we conducted this retrospective cohort study pooling data from two previously-published randomized clinical trials involving TKA patients [13,14]. These two sub-studies were part of a larger prospective project registered under NCT01459523 (http://clinicaltrials.gov); one compared proximal and distal ACC [14], and the other compared long-axis and short-axis ultrasound imaging for in-plane FNC insertion [13].

\section{Original study criteria and outcomes}

The inclusion and exclusion criteria were the same for both studies. Adults ( $\geq 18$ years of age) scheduled for unilateral knee arthroplasty with a perineural catheter planned for postoperative analgesia were assessed for inclusion. We excluded patients unable to understand the study protocol or care for the infusion pump/catheter system, and those with any known contraindication to the study medications or regional anesthesia, insulin-dependent diabetes mellitus, neuropathy of any etiology in the affected extremity, hepatic or renal failure, any additional surgical site outside of the limb intended for catheter placement, chronic opioid use, active illicit substance abuse, pregnancy, or inability to communicate with the investigators and hospital staff.

Preoperatively, all patients underwent perineural catheter insertion (Arrow FlexTip Plus, Research Triangle Park, NC, USA) under ultrasound guidance at either the femoral nerve or adductor canal location. ACCs were placed adjacent to the saphenous nerve lateral to the superficial femoral artery in the subsartorial tunnel just distal to the apex of the femoral triangle (considered "proximal" [14]) or at the mid-thigh, halfway between the anterior superior iliac spine and the top of the patella as described by Lund et al. [6].

The intraoperative anesthesia technique has not been strictly standardized, but all subjects enrolled in the previous studies $[13,14]$ received general anesthesia, combining inhalational and intravenous agents, without additional neuraxial block. All patients received the following standardized perioperative multimodal analgesic regimen designed for TKA: surgeon-administered periarticular injections of ropivacaine $0.2 \%(150 \mathrm{ml})$ and epinephrine $(2.5 \mu \mathrm{g} / \mathrm{ml})$, with ketorolac (30 mg) divided equally within the posterior capsule, retinacular layer, and subcutaneous tissue at the conclusion of surgery $[16,17]$; perineural infusion of ropivacaine $0.2 \%$ (basal rate of $6 \mathrm{ml} / \mathrm{h}$; patient-controlled bolus of $5 \mathrm{ml}$; 30-minute lockout interval); and scheduled oral oxycodone and acetaminophen tablets (5 and $325 \mathrm{mg}$, respectively) four times daily, oral diclofenac $(50 \mathrm{mg})$ twice daily, and additional oral oxycodone (5-10 mg) and intravenous (IV) nurseadministered opioid (morphine [1-2 $\mathrm{mg}$ ] or hydromorphone [0.2-0.4 mg]) as needed every 2-3 hours for breakthrough postoperative pain inadequately treated with the perineural ropivacaine infusion/bolus and oral agents.

The only difference among groups was the location of the perineural catheter. The primary outcome of these original studies $[13,14]$ was the onset time of anesthetic block in minutes. Other measurements collected on POD 1 included: average and worst post-surgical pain (NRS, 0-10), opioids consumed (mg oxycodone and/or IV morphine), number of awakenings due to 
pain, satisfaction with pain control (measured on a Likert scale; $0=$ not at all satisfied, $10=$ extremely satisfied), leakage of fluid from the catheter site, and subjective global rating of numbness (lack of sensation) in the operative extremity ( $0=$ normal sensation; 10 = completely insensate).

The primary outcome of the present study was patientreported numbness score on POD 1 . Secondary outcomes included total daily ambulation distance (meters) for POD 1, in addition to other measures collected at the time of the original studies mentioned previously. All data from all subjects who completed the study were included in the analyses. Descriptive statistics were performed, and normality was assessed by the Kolmogorov-Smirnov test. For normally-distributed data, single comparisons between the ACC and FNC groups were performed using Student's t-test; for non-normal continuous data, the Mann-Whitney $\mathrm{U}$ test was used. The $\mathrm{Z}$ test or Fisher's exact test were used for comparisons of categorical data. As a secondary analysis, we examined the association between total daily ambulation distance and numbness for POD 1 using ordinary least-squares linear regression, adjusting for age, body mass index, and ASA status. NCSS-PASS (Kaysville, UT, USA) and STATA (ver. 12.1; STATA Corp., College Station, TX, USA) software packages were used for analyses. All P values were twosided, and $\mathrm{P}<0.05$ was considered statistically significant.

\section{Results}

The data from all 94 previous clinical trial subjects were included in this study (46 FNC and 48 ACC). The FNC group was comparable to the adductor canal group in key baseline criteria (Table 1) although we did note a higher proportion of women in the femoral group relative to the ACC group (4/42 vs. $0 / 48, \mathrm{P}=$ 0.04).

ACC patients reported lower numbness scores relative to the FNC group (median $\left[10^{\text {th }}-90^{\text {th }}\right.$ percentiles]): $0(0-5)$ vs. $4(0-10)$, $\mathrm{P}<0.01$. The ACC group also demonstrated an approximately seven-fold increase in ambulation on POD 1 relative to the FNC group (median $\left[10^{\text {th }}-90^{\text {th }}\right.$ percentiles]): $45.7(6.2-91.4)$ vs 6.1 (0-42.7), $\mathrm{P}<0.01$. No differences were noted between the two groups on our other, secondary outcomes (Table 2). While linear models showed a statistically significant association between total daily ambulation distance and numbness on POD 1 (Beta = -2.6 ; $95 \% \mathrm{CI}:-4.5,-0.8, \mathrm{P}<0.01$ ), the $\mathrm{R}$-squared was 0.1 .

\section{Discussion}

For TKA patients receiving perineural local anesthetic infusions as part of a multimodal analgesic regimen, ACC results in less self-reported numbness and facilitates improved early

Table 1. Morphometric Measures and Administrative Data

\begin{tabular}{lccc}
\hline & $\begin{array}{c}\text { Femoral } \\
(\mathrm{n}=46)\end{array}$ & $\begin{array}{c}\text { Adductor canal } \\
(\mathrm{n}=48)\end{array}$ & P value \\
\hline Age $(\mathrm{yr})$ & $65(55-82)$ & $66(58-76)$ & 0.34 \\
Female/male $(\mathrm{n})$ & $4 / 42$ & $0 / 48$ & 0.04 \\
ASA physical status & $3(2-3)$ & $3(2-3)$ & 0.52 \\
Height $(\mathrm{cm})$ & $178(163-188)$ & $178(168-185)$ & 0.68 \\
Weight $(\mathrm{kg})$ & $103(77-130)$ & $101(78-135)$ & 0.24 \\
BMI $\left(\mathrm{kg} / \mathrm{m}^{2}\right)$ & $32(27-44)$ & $32(26-41)$ & 0.90 \\
Type of surgery (n) & & & 0.99 \\
$\quad$ Total knee arthroplasty & 45 & 46 & \\
$\quad$ Unicompartmental knee arthroplasty & 1 & 2 & \\
\hline
\end{tabular}

Values are reported as medians $\left(10^{\text {th }}-90^{\text {th }}\right.$ percentiles $)$ or numbers of subjects (n), as indicated; ASA: American Society of Anesthesiologists, BMI: body mass index.

Table 2. Opioid Consumption and Patient-reported Measures on Postoperative Day 1

\begin{tabular}{lccc}
\hline & $\begin{array}{c}\text { Femoral } \\
(\mathrm{n}=46)\end{array}$ & $\begin{array}{c}\text { Adductor canal } \\
(\mathrm{n}=48)\end{array}$ & P value \\
\hline Opioid consumption (mg) & $1.3[4.8]$ & $1.5[3.6]$ & 0.89 \\
Average pain (NRS 0-10) & $3(0-7)$ & $4(0-8)$ & 0.34 \\
Worst pain (NRS 0-10) & $6(2-10)$ & $10-10)$ & 0.67 \\
Satisfaction (Likert 0-10) & $10(8-10)$ & $10(8-10)$ & 0.97 \\
Awakenings due to pain (n) & $3.0[3.8]$ & $1.9[3.5]$ & 0.17 \\
\hline
\end{tabular}

Values are reported as medians $\left(10^{\text {th }}-90^{\text {th }}\right.$ percentiles $)$ or means [standard deviation], as indicated, NRS: numeric rating scale $(0-10 ; 0=$ no pain, $10=$ worst pain), n: number of events. 
ambulation when compared to FNC with no differences in pain control. Few other studies involving perineural catheters have focused on numbness as a procedure-specific outcome after surgery [18-20]. While self-reported measures such as the Western Ontario McMaster Osteoarthritis Index exist and are commonly used for long-term postoperative evaluation, these measures tend to be global, incorporating multiple dimensions such as overall well-being and independence [21,22] and have not been correlated with actual physical function. To better assess the role of regional analgesia, researchers have advocated for more clinically relevant patient-reported outcome measurement tools applicable to TKA patients [23].

Perineural catheter location is an important factor determining patient-reported numbness beyond type of local anesthetic, dosing strategy, and duration of local anesthetic infusion [24]. The 4-point median difference in numbness between the ACC and FNC groups is notable given that half of the ACC patients reported no numbness, and ACC has been previously shown to better preserve quadriceps muscle strength $[11,12]$. Patient-reported outcomes related to TKA have focused primarily on pain intensity [23], but the present study provides evidence that selfreported numbness using NRS may be easily incorporated into the bedside assessment to provide additional information. This is a clinically important outcome because the patients' subjective feeling of numbness may decrease their willingness to participate in physical therapy; physiologically, loss of proprioception and motor weakness contribute to gait and balance problems that can affect ambulation [25].

However, in our secondary analysis, although there was a significant association between total ambulation distance and numbness, $90 \%$ of the variance remained unexplained; therefore, self-reported numbness cannot be used to predict ambulation achievement. The remaining unexplained variance emphasizes the need for further research into the factors that potentially contribute to postoperative ambulation after TKA. These results are consistent with prior reports suggesting that patientreported outcomes correlate poorly with performance-based outcomes [23,26]. Furthermore, we found no group differences in satisfaction, pain experience, or opioid consumption. Since patients' own perceptions regarding their functional ability and physical status may differ greatly from their actual performance outcomes when measured, our data reinforce the need for both performance-based and self-reported outcomes when assessing the progress of TKA patients' postoperative rehabilitation [27].

Our study had several limitations that should be considered when interpreting the results. First, we used a retrospective study design such that the reported associations may not be true causal relationships; our study also pooled data from two separate clinical trials that were not powered to test for the present study's primary outcome, even though both studies measured it. We believe that the fact that we combined data from two studies may explain the difference in number of female patients per group, because randomization was not applied across the entire sample. However, a potential advantage of our study is that, although it was retrospective, it used prospectively collected data during the immediate postoperative period when the effects of the ACC and FNC techniques on numbness would be most likely to be found using the same standardized study methodology and case report forms. Although selection bias remains possible, all patients were treated on the same clinical pathway for TKA, in which only the placement location of CPNB varied. Second, the generalizability of the study results is limited to practices employing similar techniques and equipment to those used in this study, and may not be generalizable to other institutions or non-veteran populations.

In summary, although perineural local anesthetic infusion in the adductor canal results in improved early ambulation distance and lower patient-reported numbness after TKA compared to femoral perineural infusion, the correlation between these two variables was weak. Future studies evaluating the comparative effectiveness of regional analgesic techniques in TKA patients should include both patient-reported and performance-based outcome measurements.

\section{References}

1. Hebl JR, Kopp SL, Ali MH, Horlocker TT, Dilger JA, Lennon RL, et al. A comprehensive anesthesia protocol that emphasizes peripheral nerve blockade for total knee and total hip arthroplasty. J Bone Joint Surg Am 2005; 87 Suppl 2: 63-70.

2. Chelly JE, Greger J, Gebhard R, Coupe K, Clyburn TA, Buckle R, et al. Continuous femoral blocks improve recovery and outcome of patients undergoing total knee arthroplasty. J Arthroplasty 2001; 16: 436-45.

3. Jaeger P, Grevstad U, Henningsen MH, Gottschau B, Mathiesen O, Dahl JB. Effect of adductor-canal-blockade on established, severe postoperative pain after total knee arthroplasty: a randomised study. Acta Anaesthesiol Scand 2012; 56: 1013-9.

4. Ilfeld BM, Duke KB, Donohue MC. The association between lower extremity continuous peripheral nerve blocks and patient falls after knee and hip arthroplasty. Anesth Analg 2010; 111: 1552-4.

5. Feibel RJ, Dervin GF, Kim PR, Beaule PE. Major complications associated with femoral nerve catheters for knee arthroplasty: a word of caution. J Arthroplasty 2009; 24(6 Suppl): 132-7. 
6. Lund J, Jenstrup MT, Jaeger P, Sørensen AM, Dahl JB. Continuous adductor-canal-blockade for adjuvant post-operative analgesia after major knee surgery: preliminary results. Acta Anaesthesiol Scand 2011; 55: 14-9.

7. Shah NA, Jain NP. Is continuous adductor canal block better than continuous femoral nerve block after total knee arthroplasty? Effect on ambulation ability, early functional recovery and pain control: a randomized controlled trial. J Arthroplasty 2014; 29: 2224-9.

8. Jaeger P, Zaric D, Fomsgaard JS, Hilsted KL, Bjerregaard J, Gyrn J, et al. Adductor canal block versus femoral nerve block for analgesia after total knee arthroplasty: a randomized, double-blind study. Reg Anesth Pain Med 2013; 38: 526-32.

9. Mudumbai SC, Kim TE, Howard SK, Workman JJ, Giori N, Woolson S, et al. Continuous adductor canal blocks are superior to continuous femoral nerve blocks in promoting early ambulation after TKA. Clin Orthop Relat Res 2014; 472: 1377-83.

10. Charous MT, Madison SJ, Suresh PJ, Sandhu NS, Loland VJ, Mariano ER, et al. Continuous femoral nerve blocks: varying local anesthetic delivery method (bolus versus basal) to minimize quadriceps motor block while maintaining sensory block. Anesthesiology 2011; 115: 77481.

11. Kwofie MK, Shastri UD, Gadsden JC, Sinha SK, Abrams JH, Xu D, et al. The effects of ultrasound-guided adductor canal block versus femoral nerve block on quadriceps strength and fall risk: a blinded, randomized trial of volunteers. Reg Anesth Pain Med 2013; 38 : $321-5$.

12. Jaeger P, Nielsen ZJ, Henningsen MH, Hilsted KL, Mathiesen O, Dahl JB. Adductor canal block versus femoral nerve block and quadriceps strength: a randomized, double-blind, placebo-controlled, crossover study in healthy volunteers. Anesthesiology 2013; 118: 409-15.

13. Mariano ER, Kim TE, Funck N, Walters T, Wagner MJ, Harrison TK, et al. A randomized comparison of long-and short-axis imaging for in-plane ultrasound-guided femoral perineural catheter insertion. J Ultrasound Med 2013; 32: 149-56.

14. Mariano ER, Kim TE, Wagner MJ, Funck N, Harrison TK, Walters T, et al. A randomized comparison of proximal and distal ultrasoundguided adductor canal catheter insertion sites for knee arthroplasty. J Ultrasound Med 2014; 33: 1653-62.

15. Weinstein AM, Rome BN, Reichmann WM, Collins JE, Burbine SA, Thornhill TS, et al. Estimating the burden of total knee replacement in the United States. J Bone Joint Surg Am 2013; 95: 385-92.

16. Essving P, Axelsson K, Åberg E, Spännar H, Gupta A, Lundin A. Local infiltration analgesia versus intrathecal morphine for postoperative pain management after total knee arthroplasty: a randomized controlled trial. Anesth Analg 2011; 113: 926-33.

17. Tripuraneni KR, Woolson ST, Giori NJ. Local infiltration analgesia in TKA patients reduces length of stay and postoperative pain scores. Orthopedics 2011; 34: 173.

18. Le LT, Loland VJ, Mariano ER, Gerancher JC, Wadhwa AN, Renehan EM, et al. Effects of local anesthetic concentration and dose on continuous interscalene nerve blocks: a dual-center, randomized, observer-masked, controlled study. Reg Anesth Pain Med 2008; 33: 51825.

19. Ilfeld BM, Le LT, Ramjohn J, Loland VJ, Wadhwa AN, Gerancher JC, et al. The effects of local anesthetic concentration and dose on continuous infraclavicular nerve blocks: a multicenter, randomized, observer-masked, controlled study. Anesth Analg 2009; 108: 345-50.

20. Ilfeld BM, Loland VJ, Gerancher JC, Wadhwa AN, Renehan EM, Sessler DI, et al. The effects of varying local anesthetic concentration and volume on continuous popliteal sciatic nerve blocks: a dual-center, randomized, controlled study. Anesth Analg 2008; 107: 701-7.

21. Miner AL, Lingard EA, Wright EA, Sledge CB, Katz JN. Knee range of motion after total knee arthroplasty: how important is this as an outcome measure? J Arthroplasty 2003; 18: 286-94.

22. Swiontkowski MF, Engelberg R, Martin DP, Agel J. Short musculoskeletal function assessment questionnaire: validity, reliability, and responsiveness. J Bone Joint Surg Am 1999; 81: 1245-60.

23. Mizner RL, Petterson SC, Clements KE, Zeni JA Jr, Irrgang JJ, Snyder-Mackler L. Measuring functional improvement after total knee arthroplasty requires both performance-based and patient-report assessments: a longitudinal analysis of outcomes. J Arthroplasty 2011; 26: 728-37.

24. Ilfeld BM, Moeller LK, Mariano ER, Loland VJ, Stevens-Lapsley JE, Fleisher AS, et al. Continuous peripheral nerve blocks: is local anesthetic dose the only factor, or do concentration and volume influence infusion effects as well? Anesthesiology 2010; 112: 347-54.

25. Munin MC, Rudy TE, Glynn NW, Crossett LS, Rubash HE. Early inpatient rehabilitation after elective hip and knee arthroplasty. JAMA 1998; 279: 847-52.

26. Carli F, Clemente A, Asenjo JF, Kim DJ, Mistraletti G, Gomarasca M, et al. Analgesia and functional outcome after total knee arthroplasty: periarticular infiltration vs continuous femoral nerve block. Br J Anaesth 2010; 105: 185-95.

27. Bernucci F, Carli F. Functional outcome after major orthopedic surgery: the role of regional anesthesia redefined. Curr Opin Anaesthesiol 2012; 25: 621-8. 